\title{
LA VIOLENCIA DE GÉNERO COMO CAUSA DE ALGUNOS FENÓMENOS MIGRATORIOS EN EUROPA
}

\author{
Gender-Based Violence As A Cause Of Certain Migratory Phenomena In Europe
}

Lavinia BELEGGIA*

\begin{abstract}
Sumario:
Sin duda, la cuestión de género es un elemento constante de debate y lucha diaria. Hemos pasado de una presencia incesante y oclusiva del patriarcado en la vida de cada sujeto femenino, a una acción más velada pero aún incisiva. Una acción totalmente humana, como la ley y la necesidad de moverse, para crear una mejor existencia, se ha convertido en un ejemplo más de prevaricación de un tipo sobre el otro y de formas de violencia y vulnerabilidad. El análisis del fenómeno migratorio actual desde una perspectiva socio-legal es fundamental: los genocidios de los Balcanes y del África subsahariana, antes de los tratados internacionales de hoy, han creado los cimientos de la protección humanitaria, formando una rica jurisprudencia de los Tribunales Internacionales.
\end{abstract}

Resumen: El estudio de la identidad y el rol de género que ha interesado el análisis crítico de los autores internacionales autorizados del siglo pasado (De Beauvoir, Friedan, Witting, Irigaray, Kristeva, Millett, Foucault, Pitch, Butler, MacKinnon, Fineman, Nussbaum) es punto de apoyo conceptual para entender cómo, incluso hoy, el problema de la desigualdad de género es predominante en la esfera pública y privada. Con un enfoque socio-antropológico y jurídico, se vuelven evidentes los legados históricos de un "modus operandi" discriminatorio y violento de dominación del sujeto femenino, que se ve obligado a emigrar por una vana esperanza de salvación $y$ disfrute de sus derechos.

Palabras clave: Crítica de género, autodeterminación, desplazamiento-desplazamiento, normativización de la violencia de género, estudios de caso, el problema del flujo migratorio europeo desde una perspectiva de género.

Abstract: The study of identity and the role of gender that has interested the critical analysis of authorized international authors of the last century (De Beauvoir, Friedan, Witting, Irigaray, Kristeva, Millett, Foucault, Pitch, Butler, MacKinnon, Fineman, Nussbaum) it is a point of support or conceptual to understand how, even today, the problem of gender inequality is a constant predominant in the public and private sphere. With a socio-anthropological and legal approach, the historical legacies of a discriminatory and violent "modus operandi" of the domination of the female subject, who are forced to emigrate by a vain hope of salvation and enjoyment of their rights, become evident.

\footnotetext{
* Graduada en derecho en 2018 en la Universidad de Bolonia en Italia con una tesis en filosofía del derecho intitulada "El gobierno del cuerpo y su explotación: Análisis filosófico legal de algunos escenarios contemporáneos”. En marzo recibiré el título de Maestra después de la conclusión del máster en migración y desarrollo internacional con una tesis que se intitulada "La aparición de los sumergidos en el fenómeno de la trata de personas. Vuelva a leer y reflexiones sobre la esclavitud sexual de las mujeres en el Este de Europa. Una propuesta antropológica". Después de la conclusión de una fase en el Departamento de Igualdad de Oportunidades de la Municipalidad 1a de Roma (Sportello Roxanne). lavinia_beleggia@libero.it
} 
Keywords: Criticism of gender, self-determination, displacement-displacement, normativization of gender violence, case studies, the problem of the European migratory flow from a gender perspective.

Con la tesis titulada "El gobierno del cuerpo y su explotación: Análisis filosófico y jurídico de algunos escenarios contemporáneos", defendida en marzo de 2018 en la Facultad de Derecho del Alma Mater Studiorum de Bolonia, busqué entender cómo la visión del cuerpo ha sido objeto de diversas teorizaciones que han determinado la evolución de su usabilidad y autodeterminación en diversos ámbitos.

Como introducción al estudio del fenómeno migratorio que tiene como sujeto a la mujer es fundamental analizar cómo su existencia ha sido objeto de innumerables protestas y luchas, a fin de modificar profundamente la visión social e históricamente impuesta; en particular, su cuerpo y habilidad para actuar.

Retrotrayendo la evolución del pensamiento feminista en la ley (feminismo liberal, igualdad, diferencia, teoría radical, teoría queer y el post feminismo) se evidencia cómo los paradigmas teóricos de referencia, en particular la crítica de género, han servido para obtener un marco básico para notar cómo la persona, su entidad externa e interna, está en prima facie, separada de los dogmas de subyugación ${ }^{1,2}$ y heteronormatividad ${ }^{3}$.

Las perspectivas feministas se han dedicado a la crítica del positivismo como una ciencia objetiva que neutraliza la subjetividad, dividiendo el sujeto en una clave científica, en dos entidades sexuales. La crítica se mueve en la deconstrucción de los significados preestablecidos ${ }^{4}$ y objetivos de la ciencia, a fin de reapropiarse de una realidad que tiende al particularismo y su autodeterminación.

El cuerpo, en particular el femenino, ha estado en la realidad histórica moderna sujeto a actitudes de dominación social ${ }^{5}$, de marcación de género y de la circunscripción de su poder ${ }^{6}$. Solo a través de una nueva conciencia ha logrado liberarlo de la discriminación y del menosprecio histórico. Nos enfrentamos a un envoltorio experiencial cuyo titular tiene la libertad de utilizarlo y exigir su pleno respeto.

Las diferencias de género y, por lo tanto, de rol y temperamento derivan de creaciones culturales seculares: las estandarizaciones resultantes determinan la creación de dos personalidades,

${ }^{1}$ El lenguaje adquiere el poder de crear la realidad social a través de los actos de comunicación de los sujetos que hablan. Butler, Judith (2013), Cuestión de género. El feminismo y la subversión de la identidad, Laterza, Roma, p. 163 .

2 Manojos de realidad sobre el cuerpo social. Witting, Monique (1976), El cuerpo lésbico, Edizioni delle Donne, Roma, p. 4.

3 La unidad de género es el efecto de una práctica normativa que busca estandarizar la identidad de género a través de la heterosexualidad obligatoria. El hecho de que los regímenes de poder del heterosexismo y el falocentrismo intenten incrementarse a través de una constante repetición de su lógica, metafísica y ontologías naturalizadas, no implica que la repetición sea interrumpida, como si realmente pudiera interrumpirse, pero modificada.

4 El sexismo, la columna vertebral de la sociedad androcéntrica, es la forma de racismo más extendida, pero menos reconocida y cuestionada. RiverA, Annamaria (2010), Lo bello, la bestia y lo humano, Ediesse, Roma, p. 35.

5 El cuerpo como medio pasivo o instrumento que espera ser animado por una voluntad mayoritariamente inmaterial. Butler, Judith (2013), op. cit., nota 1, p. 15.

${ }^{6}$ El Logos estructura por exclusión aquella equivalencia que le permite designar su propio campo, renunciando a todos los demás y asignando el significante y el significado a un sistema de control recíproco. GALIMBERTI, Umberto (2002), El cuerpo, Feltrinelli, Milan, p. 374. 
femenina y masculina, contrastantes y antitéticas, pero al mismo tiempo complementarias para la creación de esa homologación creada por la propia institución y desde el reacondicionamiento de los dos sexos a funciones fisiológicas y éticas bien establecidas. No habrá más espacio para la versatilidad y la identidad subjetiva de cada ser, de las cualidades naturales de estos seres.

La insistencia en la inexistencia de diferencias entre los sexos, en una sociedad que siempre ha creído en estas diferencias y en ellas ha sido modelada, puede ser una forma de estandarización de la sociedad tan sutil tanto como la de insistir en la existencia de muchas diferencias. [...] si abolir las diferencias entre las personalidades reconocidas del hombre y la mujer significa abolir toda forma de expresión del tipo de personalidad que se consideraba exclusivamente femenina o exclusivamente masculina, entonces esta solución implica una pérdida social. [...] Donde hoy no tenemos esos modelos de comportamiento para las mujeres y modelos de comportamiento para los hombres, entonces tendríamos muchos modelos de comportamiento, que expresarían los intereses de muchas personas con tantas categorías diferentes de talentos. [...] Sería necesario conocer toda la gama de potenciales humanos, y con ella fabricar, un tejido social menos arbitrario, en el que cada dote humana diferente encuentre el lugar que le convenga ${ }^{7}$.

El enfoque jurídico de la identidad del cuerpo ve una evolución continua de las separaciones, superposiciones, compensaciones y composiciones: es necesario reequilibrar el antropocentrismo con el biocentrismo para comprender y conocer mejor la investigación más íntima de la identidad personal.

El ius es necesario pero no suficiente para establecer esta relación, en cuanto que debe liberar a los seres para encontrar su propio espacio en el mundo, eliminando los elementos moralmente cargados (evitar la ética del Estado) y abrir la autonomía de acción para cada sujeto. Salvar la acción correcta de la política y la ética es el principal baluarte para un jurista moderno y para la misma sociedad. Es necesario entender cómo es un error común reemplazar el derecho a la moralidad como el único observatorio de referencia para problematizar las ciencias de la vida, pero para abrirse a las muchas posibilidades en juego.

La evolución jurisprudencial y legislativa italiana ${ }^{8}$ se ha convertido en la voz de varias reinterpretaciones sobre el sentido de pertenencia corporal: hemos pasado de una visión para la cual la

7 Mead, Margaret (2009), Sexo y temperamento en tres sociedades primitivas, Il saggiatore, Milan, pp. 328-329-334.

8 En el contexto italiano, desde los años setenta hasta los primeros años de 200o, varias intervenciones legislativas han influido significativamente en la esfera de los derechos individuales y la transformación de áreas cruciales de la vida social, como en la realidad familiar. Gazzetta Ufficiale della Repubblica Italiana, Ley 1 de diciembre de 1970, $n 898$ - "Disciplina de los casos de disolución del matrimonio", [en linea], disponible en: http://www. gazzettaufficiale.it/eli/id/1970/12/o3/o7oUo898/sg;jsessionid=Y2ITexX-cjfTXYBT4MhBqQ_.ntc-as3-guri2b (la llamada ley de Fortuna-Baslini), derechos de procreación e interrupción. Gazzetta Ufficiale Della RepubBlica ItAliana, Ley 22 de mayo de 1978, n.194, [en linea], disponible en: http://www.gazzettaufficiale.it/atto/ serie_generale/caricaDettaglioAtto/originario?atto.dataPubblicazioneGazzetta=1978-05-22\&atto.codiceRedazi onale $=078$ Uo194\&elenco3ogiorni $=$ false.

Reglas para la protección social de la maternidad e interrupción voluntaria de la embarazo, procreación con asistencia médica. Gazzetta Ufficiale della Repubblica Italiana, Ley No 40 de 19 de febrero de 2004 [la ley también se conoce como ley 40 o ley 40/2004], [en linea], disponible en: http://www.gazzettaufficiale.it/eli/ $\mathrm{id} / 2017 / 02 / 17 / 17 \mathrm{Gooo} 24 / \mathrm{sg}$.

Normas sobre la procreación con asistencia médica, normas sobre donación de órganos y trasplantes. GAzZETTA Ufficiale della Repubblica Italiana, Ley 1 de abril de 1999, No. 91, [en linea], disponible en: http://www. camera.it/parlam/leggi/99o91l.htm.

Decreto Ministerial del 8 de abril de 200o, actualizado con el Decreto Ministerial del 11 de abril de 2008 y declaraciones antes de la muerte. (Ley 22 de diciembre de 2017, No. 219-Normas en consentimiento informado y disposiciones de tratamiento anticipado). 
prohibición de la disposición del propio cuerpo es absoluta ${ }^{9}$ a una apertura hacia la realización de cómo el sujeto es, él mismo, en todas sus facultades, autor de una posible modificación de su corporeidad, como parte de su esfera de autodeterminación y libertad personal (la libertad de disponer de su cuerpo es la explicación de autodeterminación en las elecciones personales relacionadas con el perfil corporal, incluso con lesiones físicas aparentes pero funcionales al equilibrio psico-físico $)^{10}$.

En la esfera de lo que me pertenece, encuentro mi cuerpo que se destaca de todos los demás por una particularidad única: es el único cuerpo que no solo es un cuerpo, sino mi cuerpo; el cuerpo solo, dentro del estado abstracto cortado por mí en el mundo al cual, según la experiencia, yo coordino, de diferentes maneras, campos de sensación; es el cuerpo del que dispongo de modo inmediato como dispongo de sus órganos ${ }^{11}$.

Concebir un poder de control y dominio sobre el cuerpo y sus partes nos mueve de una visión instrumental de él a concebirlo como un envoltorio experiencial: La pertenencia afirma la identidad, circunscribe el cuerpo vivo, el cuerpo-yo mismo; refiriéndose a partes del cuerpo, encuentra el significado que lleva en a la raíz, sea parte de un todo que es el ego percibido: Como alma, la identidad es tota in toto y en una sola parte, pars es un lugar del self ${ }^{12}$.

Hay muchos eventos históricos que tienen como elemento fundamental la relación del cuerpo con el género y cómo este último se manifiesta como un vehículo para realizar acciones inhumanas y en contra de la dignidad.

En la segunda mitad de la década de 1990, los conflictos étnicos en la ex-Yugoslavia y Ruanda fueron un ejemplo de cómo la violencia de género en situaciones de conflicto armado fue aceptada tácitamente como inevitable para concretar, simbólicamente, el diseño macabro del grupo dominante: limpieza racial utilizando los cuerpos de mujeres y sujetos vulnerables ${ }^{13}$.

$\mathrm{Al}$ analizar el contexto histórico, en el país de África oriental (Ruanda), la división étnica y antropológica que determinó el conflicto interno se remonta a las conquistas alemanas y belgas del período colonial (1920), así como a la presencia de misiones religiosas asignadas por estos

9 Art.5 cc- "Actos de disposición del propio cuerpo": Los actos de disposición del propio cuerpo están prohibidos cuando causan una disminución permanente de la integridad física, o cuando son contrarios a la ley (579 Código Penal), al orden público o la moralidad (32 Constitución).

${ }^{10}$ A través de la lectura sinérgica de los Artículos fundamentales, que se puede encontrar en la Constitución italiana, y el trabajo jurisprudencial de los diversos tribunales italianos, fueron las sentencias destinadas a un pleno reconocimiento de la autodeterminación y la disponibilidad física: Sentencia del Tribunal Constitucional n. 221/2015 sobre el tema del cambio de sexo; Tribunal de Roma, enviado. n. 2049/2007, Caso de Welby: no hay lugar para proceder contra el médico que ha interrumpido el tratamiento de soporte vital.

${ }^{11}$ Husser L, Edmund (1999), Meditaciones cartesianas. Introducción a la fenomenología, Kluwer Academic Publishers, Berlin, p. 119.

${ }^{12}$ RodotÀ, Stefano y Zatti, Paolo (eds. 2011), Tratado de biodiritto. El gobierno del cuerpo, (vol.I-II), Giuffrè Editore, Milan, p. 113 .

${ }^{13}$ El mundo de la ley ha creado implícitamente una subdivisión abstracta, enfatizando el tema de la ley normal porque está contenida en ese conjunto de elementos concretos (adulto, blanco, alfabetizado, propietario) que lo hacen disfrutar plenamente de sus capacidades, con un "minimalismo de derechos que tiende a simplificar la complejidad de esta semántica de desigualdad, centrándose exclusivamente en la categoría de libertad y, además, solo en su dimensión negativa, y en el criterio de la capacidad de actuar, aboliendo efectivamente la discusión sobre la libertad positiva, sobre la dignidad y lo que a menudo permanece encubierto, la posibilidad misma de enmarcar figuras subjetivas expresivas de la condición humana, de su multiformidad, así como su fragilidad. CASADEI, Thomas (2012), Derechos humanos y personas vulnerables. Violaciones, transformaciones, aporias, Giappichelli Editore, Torino, p. 96. Se crea un mecanismo de exclusión para la garantía y la protección ilusoria, para lo que se excluye a los no capaces y, por lo tanto, débiles, indefensos y vulnerables, no se pueden referir a la normalidad; 
países, que produjeron una mayor brecha de identidad entre los hutus (aquellos que, según las creencias populares, fueron encontrados en la tierra y por su origen vinculado al cultivo) y los tutsi (los caídos del cielo, grandes criadores de ganado) y el twa, los tres grupos étnicos más relevantes.

Los misioneros encargados de ayudar a la población (1950) manipularon los mitos autóctonos: el linaje hamita ${ }^{14} \mathrm{y}$ este uso genealógico se utilizó durante mucho tiempo para discriminar las cepas africanas como sucesores del sujeto desheredado y dotados de ciertos atributos físicos y de carácter. Fue después de estudios científicos que la raza de los sobrevivientes de la humanidad original (bereberes, egipcios, abissini) y las características físicas de los tutsi —altos, potentes pero delgados - y su pigmentación los elevaron a una consideración más positiva que sus cohabitantes ${ }^{15}$.

Esta conceptualización le dio a los tutsi la oportunidad, durante el régimen colonial belga, de ocupar cargos administrativos prominentes, aumentando la división étnica interna y causando el resentimiento de los hutu hacia ellos.

El establecimiento de un monopolio político a favor de los primeros es la hija de la ficción colonial $^{16}$ : el grupo étnico y el conjunto de elementos objetivos y subjetivos, específicamente elementos subjetivos objetivados, fueron creados en la mesa por los actores europeos para desarmar la identidad individual y un grupo social cohesionado. También el simbolismo de la revuelta y propaganda hutu (Partido Revolucionario Hutu) es un ejemplo de la definición de identidad en términos de pertenencia étnica (denigración total del Otro-Tutsi): las formas de matar reflejan alegóricamente el origen de la diferencia de clase y la pertenencia a una determinada actividad, por ejemplo, los hutu, siempre cultivadores y expertos en cortar los árboles

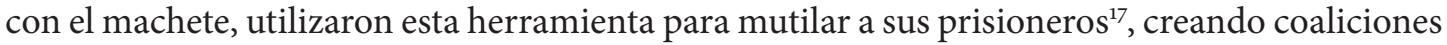
listas para matar a gran escala y contextualizando el proceso de redención de identidad de una parte de la sociedad ruandesa que se consideraba constantemente amenazada por una cultura de violencia y crueldad, denigración y desigualdad ${ }^{18}$.

A fines de agosto de 1994, el ACNUR había estimado más de dos millones de refugiados en los países vecinos, de los cuales 1, 2 millones se encotraban en Zaire, 580 mil en Tanzania, 270 mil en Burundi y 10 mil en Uganda. A fines de 1994, la crisis de Ruanda ya había costado millones de vidas. Además de las 800 mil víctimas del genocidio y los dos millones de refugiados

\footnotetext{
es un fenómeno estructural de opresión y limitación de algunos grupos de personas. Necesitamos un enfoque subversivo de los derechos. Ibidem, p. 111.

${ }^{14} \mathrm{Ham}$, en las escrituras cristianas y hebreas, es el hijo pecaminoso de Noé, que creó la dinastía de la piel oscura.

15 "La etnología de Génesis", Chrétien, Jean-Pierre (1977), Les deux visages de Cham. Points de vue français du XIXe siècle sur les races africaines d'après l'exemple de l'Afrique orientale, en GuIRAL, P. y Temine, E., L'idée de race dans la pensée politique française contemporaine, Paris, C.N.R.S., française, Editions du CNRS, pp. 177-99 y 187.

${ }^{16}$ Fusaschi, Michela (200o), Hutu-tutsi. En los orígenes del genocidio ruandés, Boringhieri, Torino, p. 121.

${ }^{17}$ El verbo gutema, que se traduce como "cortar", se refiere a una serie de actividades relacionadas con el cultivo, queridas por los hutu: para matar a los tutsi es necesario cortar y cortar los vínculos que tienen con el territorio nacional. Esta interpretación es hija de la demagogia propagandística de la revolución con alegorías etnográficas: Se cree que los tutsis son de origen etíope, por lo tanto, extranjeros, no pueden vivir con hutu, ni siquiera son físicamente similares a ellos, son demasiado altos: por lo tanto, es necesario acortarlos para emplear una expresión del período. Ibidem, p. 150.

${ }^{18}$ El evento (6 de abril de 1994) que desencadenó el genocidio de Ruanda fue el derribo del avión con misiles que transportaban a los presidentes de Ruanda y Burundi después de asistir a una cumbre para contrarrestar las dificultades étnicas que afectaban a las naciones. La paternidad del ataque nunca fue declarada oficialmente, pero se presume que se atribuye a las falanges extremistas de la Guardia Presidencial, la mayoría hutu, siempre contraria a la colaboración del gobierno con la minoría étnica.
} 
fuera de Ruanda, aproximadamente 1,5 millones de personas fueron desplazadas en el propio país. De una población de siete millones, más de la mitad había sufrido directamente las consecuencias del conflicto.

Las mujeres son los sujetos que más sufrieron el peso del conflicto: infibulados y violados, fueron abandonados por la comunidad de pertenencia o vendidos para obtener un breve respiro de la hegemonía de los grupos contingentes.

Con respecto a los antecedentes históricos del conflicto armado en la ex-Yugoslavia, la República Federal Socialista de Yugoslavia, establecida el 29 de noviembre de 1943 por el mariscal Tito, estaba integrada por seis estados: Croacia, Serbia, Eslovenia, Bosnia-Herzegovina, Montenegro, Macedonia y dos regiones autónomas, unidas con Serbia (Kosovo y Vojvodina).

En los años 8o, con la muerte de Tito, el colapso estatal y económico comenzó y terminó con la declaración de independencia de muchos Estados por parte de la Federación Yugoslava: la primera fue Eslovenia, en 1991, con la «Guerra de los diez días» y la repentina respuesta antagónica a este movimiento independentista de Serbia, que terminó con la aceptación del nuevo Estado; luego se movió Croacia, pero en este caso el conflicto fue más largo debido a una fuerte presencia serbia dentro de la población que presionaba para que el Estado permaneciera dentro de la Federación (Guerra Serbo-Croata); mientras que Bosnia y Herzegovina, considerado un microcosmos étnico debido a la presencia de varios grupos étnicos mezclados (croatas, católicos, serbios, ortodoxos, bosnios, musulmanes) estaba bajo el control de los dos Estados vecinos, Croacia y Serbia, que tendían a invadirlo para unificar los territorios, en función de la presencia étnica de referencia.

Desde 1989, en Kosovo se ha producido una situación de discriminación total por parte de los serbios contra la población albano-kosovar (acceso denegado a puestos de trabajo y servicios públicos y al ejercicio de los derechos fundamentales). Entre 1989 y principios de 1998, se estima que 350 mil albano-kosovares abandonaron la provincia, principalmente hacia países de Europa occidental ${ }^{19}$.

Con el boicot del referéndum de 1992 por los serbios (el 90\% de la población había votado a favor de la independencia) se proclamó la independencia del Estado, pero al mismo tiempo, los serbios de Bosnia crearon, con el apoyo del presidente serbio Milošević, la República Serbia de Bosnia (el 70\% del territorio del país bosnio), encabezado por el psiquiatra Radovan Karadžićco y el general Ratko Mladić ${ }^{21}$ (defensores de las milicias serbias de Bosnia, los chetniks), miembros del partido del presidente serbio. Desde ese momento hasta 1995 la capital, Sarajevo, estuvo bajo sitio, teatro de violencia inhumana (se cuentan 181 mil víctimas en el territorio bosnioherzegoviniano ${ }^{22}$ ).

En 1993, los croatas también decidieron establecer un estado autónomo dentro de Bosnia, rompiendo las relaciones ya lábiles con la población de origen musulmán bosnio y creando un clima de terror y violencia: el objetivo unívoco de los dos

\footnotetext{
${ }^{19}$ UNHCR, Guerra y acción humanitaria: Irak y los Balcanes (2014), [en linea], disponible en: http://www.unhcr. org/pubs/sowr20oo/italian/cho9.pdf

20 Tribunal Penal Internacional por la antigua Yugoslavia, Acusaciones contra Radovan Karadzic y Ratko Mladic, (24 julio 1995), [en linea], disponible en: http://www.icty.org/x/cases/karadzic/ind/en/kar-ii950724e. pdf

${ }^{21}$ Idem.

${ }^{22}$ Idem.
}

CienCIA JuRÍdica. Departamento de Derecho. División de Derecho, Política y Gobierno, Universidad de Guanajuato - Año 9, No. 17, 2020 
Estados conquistadores y usurpadores era la destrucción de un Estado unido y multicultural, una limpieza étnica dirigida a eliminar el germen de los territorios bajo su jurisdicción ${ }^{23}$.

En este marco, la programación de la violación étnica tuvo como propósito el debilitamiento, la humillación y la denigración en las profundidades del grupo bosnio, especialmente en mujeres, visto, en un nivel iconográfico-simbólico, como la máxima representación de lo que el Estado debe proteger como generadores del futuro; era necesario explotar el cuerpo de mujeres para sellar la supremacía de un grupo étnico sobre el otro en virtud de antagonismos ideológicos ${ }^{24}$.

Muchas historias de quienes tuvieron la fuerza, después de la conclusión del conflicto, para contar su historia a las autoridades o grupos que apoyaron la recuperación de las víctimas, hablan de embarazos no interrumpidos porque los mismos violadores secuestraban a las víctimas para no concluir la interrupción (los hijos del odio, como fueron llamados estos niños, resultado de una violación).

Tras estos conflictos, por primera vez, con la ayuda de solicitudes de ciudadanos-víctimas y asociaciones que lucharon por una mayor protección, las Naciones Unidas capturaron las crueldades y la violencia ${ }^{25}$ que caracterizaron el período histórico: estableció los Tribunales Penales Internacionales para la exYugoslavia (TPIY-1993) ${ }^{26}$ y para Ruanda (TPIR-1994) ${ }^{27}$, cuyos estatutos reconocieron como delito contra la humanidad, la violación junto a la tortura y el exterminio, si se cometieron durante un conflicto armado y si estaban dirigidos contra una población civil como forma de genocidio ${ }^{28}$.

Estos resultados jurisprudenciales fueron decisivos para la elaboración del Estatuto de la Corte Penal Internacional (CPI), aprobado el 17 de julio de 1998; el Estatuto de Roma enumera los diversos tipos de violencia sexual además de la violación y la prostitución forzada como, por ejemplo, la esclavitud sexual, el embarazo y la esterilización forzada y otras formas de violencia

${ }^{23}$ En julio de 1995, los serbios decidieron llevar a cabo el último asalto, centrándose en los enclaves (reuniendo ciudades de los refugiados bosnio-musulmanes, protegidos por los Cascos Azules de la ONU, quienes tuvieron que desmilitarizar a estos últimos para evitar acciones de guerrilla contra los serbios), comenzando por Srebrenica. Las defensas humanitarias no hicieron nada y se cuentan diez mil personas muertas a sangre fría y arrojadas a fosas comunes. Hay 7.475 personas desaparecidas y presumiblemente muertas. Ibidem, (12 febrero 200o), [en linea], disponible en://www.icty.org/x/file/About/OTP/War_Demographics/en/krstic_srebrenica_ooo212.pdf.

${ }^{24}$ La estimación de las víctimas de violación supera los 22.0oo y según algunos llega a 50.ooo. Di PALMA, Sara Valentina (2010), "La violación como arma contra las mujeres: la ex Yugoslavia, Ruanda y el área de los grandes lagos africanos", en Flores, Marcello, Los raptos de guerra. Violencia masiva contra las mujeres en el siglo XX, Franco Angeli, Milano, p. 218.

25 Ya en el párrafo 2 del artículo 27 de la Convención de Ginebra de 1950, se afirmó que las mujeres estarán especialmente protegidas contra cualquier ofensa en su honor y, en particular, contra la violación, la coacción a la prostitución y cualquier ofensa a su pudor.

${ }^{26}$ United Nacions (25 mayo 1993), Resolucion 827/1993 Security Council, [en linea], disponible en: https://www. peacelink.it/cd/docs/1146.pdf

${ }_{27}$ Ibidem, Resolucion 955/1994 Security Council, [en linea], disponible en: http://unictr.irmct.org/sites/unictr.org/ files/legal-library/941108_res955_en.pdf

28 International Residual Mechanism for Criminal Tribunals, Casos Kayishema y AKAYESU, Jean Paul (junio 2001), [en linea], disponible en: http://unictr.irmct.org/en/cases/ictr-96-4 http://unictr.irmct.org/ en/cases/ictr-95-1 
sexual de gravedad como actos constitutivos tanto de delitos contra la humanidad ${ }^{29}$ como de crímenes de guerra cometidos en conflictos de carácter internacional (artículos 7-8).

El preámbulo del estatuto mencionado anteriormente es emblemático y describe los principios fundamentales de la responsabilidad plurilateral entre los Estados signatarios y la jurisdicción de la Corte en el manejo de controversias:

Conscientes de que todos los pueblos están unidos por estrechos lazos y sus culturas configuran un patrimonio común y observando con preocupación que este delicado mosaico puede romperse en cualquier momento, teniendo presente que, en este siglo, millones de niños, mujeres y hombres han sido víctimas de atrocidades que desafían la imaginación y conmueven profundamente la conciencia de la humanidad, reconociendo que esos graves crímenes constituyen una amenaza para la paz, la seguridad y el bienestar de la humanidad, afirmando que los crímenes más graves de trascendencia para la comunidad internacional en su conjunto no deben quedar sin castigo y que, a tal fin, hay que adoptar medidas en el plano nacional e intensificar la cooperación internacional para asegurar que sean efectivamente sometidos a la acción de la justicia, decididos a poner fin a la impunidad de los autores de esos crímenes y a contribuir así a la prevención de nuevos crímenes, recordando que es deber de todo Estado ejercer su jurisdicción penal contra los responsables de crímenes internacionales, reafirmando los Propósitos y Principios de la Carta de las Naciones Unidas y, en particular, que los Estados se abstendrán de recurrir a la amenaza o al uso de la fuerza contra la integridad territorial o la independencia política de cualquier Estado o en cualquier otra forma incompatible con los propósitos de las Naciones Unidas, destacando, en este contexto, que nada de lo dispuesto en el presente Estatuto deberá entenderse en el sentido de que autorice a un Estado Parte a intervenir en una situación de conflicto armado o en los asuntos internos de otro Estado, decididos, a los efectos de la consecución de esos fines y en interés de las generaciones presentes y futuras, a establecer una Corte Penal Internacional de carácter permanente, independiente y vinculada con el sistema de las Naciones Unidas que tenga competencia sobre los crímenes más graves de trascendencia para la comunidad internacional en su conjunto, destacando que la Corte Penal Internacional establecida en virtud del presente Estatuto será complementaria de las jurisdicciones penales nacionales, decididos a garantizar que la justicia internacional sea respetada y puesta en práctica en forma duradera [...]

El párrafo 6 del artículo 43 dicta el establecimiento de una División de asistencia a las víctimas y testigos encargados de ayudar a quienes comparezcan ante la Corte, así como la disposición de las medidas y disposiciones que deben tomarse para garantizar su protección y seguridad, y de especialistas en ayudar a víctimas de traumas después de la violencia; el artículo 68, "Protección de víctimas y testigos y su participación en el juicio", establece la posibilidad de que los procedimientos se realicen a puerta cerrada y permite que las disposiciones se realicen electrónicamente o por medios especiales y, finalmente, el art. 87, "Solicitudes de cooperación a los Estados partes”, sobre la protección de la información, para lo cual la Corte puede tomar las medidas

\footnotetext{
${ }^{29}$ En el Estatuto, el concepto de crimen de lesa humanidad se libera del contexto de conflicto armado; destaca la inclusión de la trata de seres humanos entre los crímenes de lesa humanidad como una reducción de la esclavitud y, por primera vez, los motivos de género entre aquellos basados en la persecución. Corte PenAL InTERNACIONAL, Estatuto de Roma. 1998, [en linea], disponible en: http://www.cirpac.it/pdf/testi/Statuto\%20di\%20 Roma\%2odella\%2oCorte\%2oPenale\%2oInternazionale.pdf (Artículo 7-h).
} 
necesarias para el bienestar psicofísico de la parte interesada, preservando su seguridad en cada momento del proceso.

Con tales instrumentos de derecho internacional, el Consejo de Seguridad de las Naciones Unidas ha trabajado arduamente para aumentar la concientización y delinear las líneas de acción para abordar el problema de la violencia sexual y el hostigamiento que sufren los civiles, promulgando diversas resoluciones, como la n. 1325 de 2000, instando a los Estados a intensificar la participación de las mujeres en la prevención y en la resolución de conflictos y en la consolidación de la paz y enfatizando la importancia de la participación conjunta y la plena intervención en todos los esfuerzos para mantener y promover la paz y la seguridad, y la necesidad de aumentar su papel en los procesos de toma de decisiones en la prevención y resolución de conflictos, incluidas las políticas (Planes de Acción Nacionales) y los procedimientos para proteger a las mujeres y los niños que son sensibles a los delitos de tipo sexuales con una política de tolerancia cero.

En la R. n. 1820/2008 se afirma que la violencia sexual en situaciones de conflicto armado puede constituir un crimen de guerra, un crimen de lesa humanidad y un presagio de genocidio; es necesario reconocer el vínculo entre la seguridad internacional y la violencia sexual en los casos en que se utiliza como táctica de guerra. La R. 1960/2010, que invita a los Estados signatarios a realizar compromisos específicos e indicar plazos precisos para combatir la violencia sexual, instándolos desde el lado de la prevención a prohibir tales delitos mediante la administración de órdenes precisas a las cadenas de mando y la imposición de códigos de conducta y, en el aspecto judicial, investigar los supuestos abusos, llevando sin demora a los responsables ante la justicia. El Representante Especial de la ONU para la violencia sexual en situaciones de conflicto, nombrado por el Secretario General (R. n. 1888/2009 que identifica esta figura en la coordinación del trabajo de las Naciones Unidas sobre el tema, hoy Pramila Patten), que supervisa el refinamiento de cuáles son los lineamientos de la Acción de la ONU contra la Violencia Sexual en Conflicto. Esta iniciativa une a 13 organismos de la ONU con el objetivo de poner fin a la violencia sexual en los conflictos, en un esfuerzo concertado para mejorar la coordinación y la rendición de cuentas, ampliar la planificación previa y posterior al conflicto y apoyar los esfuerzos nacionales para prevenir la Violencia sexual, respondiendo efectivamente a las necesidades de las y los sobrevivientes.

La R. n. 2106/2013 se centra específicamente en el tema de la violencia sexual en situaciones de conflicto armado. El documento agrega detalles operativos adicionales a las resoluciones anteriores sobre el tema y reitera la necesidad de esfuerzos más intensos por parte de todos los actores, no solo del Consejo de Seguridad y las partes en un conflicto armado, sino de todos los Estados miembros y entidades de las Naciones Unidas, para la ejecución de los mandatos emitidos en las resoluciones sobre el tema y por la lucha contra la impunidad de estos crímenes.

R. n. 2122/2013 que establece la obligación de reservar asientos para mujeres en las mesas de paz y en la R. n. 2242/2015, que identificó la participación de las mujeres como el factor clave para determinar las operaciones efectivas en la mediación de la paz, el mantenimiento de la paz y la prevención de conflictos.

Existen otros instrumentos legales, anteriores y contemporáneos a las resoluciones anteriores, que han sido fundamentales para aumentar la base de protección de la violencia en cualquier contexto existencial: la Declaración Universal de los Derechos Humanos (1948), el Convenio Europeo para la Protección de los Derechos Humanos y de Libertades Fundamentales (1950), la Resolución del Consejo de Seguridad n. 1973/1975, "Declaración sobre la protección de todas las personas sometidas a tortura”, (en la que se preparan programas de capacitación para 
que la policía estatal se enfrente al problema, la inserción del delito de tortura en la legislación nacional, la obligación de investigar de manera imparcial y la indemnización de las víctimas), la CEDAW (1979), la Convención contra la Tortura y otros Tratos o Penas Crueles, Inhumanos y Degradantes ( CAT-1984), la Carta Africana de los Derechos Humanos y de los Pueblos (1986), la Carta Árabe (1994), la Convención de Beijin (1995), el Convenio de Lanzarote (2007prevención y represión de todas las formas de explotación sexual), el Convenio de Estambul (2011-prevención y lucha contra la violencia de género y doméstica). Se habla de violencia de género cada vez que nos enfrentamos a un acto basado en el objetivo de causar daños físicos, sexuales, psicológicos, económicos, amenazas, coerción, privación arbitraria de la libertad, en la vida privada y pública.

Los hechos analizados anteriormente pueden considerarse un ejemplo básico para comprender y enfocar, con herramientas más apropiadas a nivel sociológico y jurídico, el impacto que los conflictos civiles han tenido en la generación de flujos migratorios que afectan la situación europea en los últimos veinte años.

La migración de individuos vulnerables varía según el cambio geocultural, la edad y el género, los niveles educativos, las necesidades económicas. La búsqueda de trabajo y la reunificación familiar, el deseo de emancipación y las represalias en contextos culturales represivos y peligrosos son las principales causas que determinan este fenómeno social. Nos enfrentamos a la necesidad universal de escapar de los estilos de vida y las condiciones que ya no se pueden compartir, de una privación constante de garantías internacionalmente reconocidas como inalienables y fundamentales para disfrutar de la plena disponibilidad de las libertades fundamentales.

En este punto de la discusión es interesante tener, para una mayor visión general del fenómeno, los datos estadísticos del flujo migratorio italiano en los últimos años: a un nivel puramente cuantitativo, los flujos registrados en Italia siempre han sido erráticos: picos a principios de los años 90 (443 de 1990 y 2 mil 254 de 1991) y una disminución significativa desde los años 1995 hasta 1999. En 2003 volvemos a calcular las solicitudes de 1987 con una subsiguiente disminución y desaparición de la demanda, que luego reaparece con fuerza en 2008 (4 mil 960) ${ }^{30}$ y disminuye con picos y descensos constantes hasta 2017 (-15\% de variación en las principales nacionalidades solicitantes entre 2016-2017) $)^{31}$ y un aumento del 55\% entre agosto y septiembre de $2018^{32}$.

Al centrarse en el análisis de una manera más amplia, es posible ver cómo el número de desembarques de migrantes en las costas italianas se ha vuelto variable, a diferencia de lo que declaran los medios de comunicación y la dialéctica política, sin tener en cuenta el hecho de que es un problema social que debe afrontarse con ímpetu y eficiencia. Comparando los datos del 1 de enero de 2018 hasta el 19 de octubre, frente a datos del mismo periodo de los años 2016 y 2017, es interesante observar que en 2016 hubo 146 mil 287 aterrizajes (-85.07\%), en 2017, 110

\footnotetext{
30 Ministerio del Interior Italiano, Cuaderno Estadístico por los annos 1990-2017, [en linea], disponible en: http://www.libertaciviliimmigrazione.dlci.interno.gov.it/sites/default/files/allegati/quaderno_statistico_per_ gli_anni_1990-2017_ok.pdf

${ }^{31}$ Ministerio Del Interior Italiano, Datos estadistico 2016-2017, [en linea], disponible en: http://www.libertaciviliimmigrazione.dlci.interno.gov.it/sites/default/files/allegati/dati_asilo_2017_.pdf

${ }^{32}$ Ibidem, Datos estadistico sptiembre 2018, [en linea], disponible en: http://www.libertaciviliimmigrazione.dlci. interno.gov.it/sites/default/files/allegati/settembre_2018_.pdf
} 
mil 636 (- 80.26\%) y en 2018, 21 mil 839, de los cuales, menores no acompañados son, en 2016, 25 mil 846, en 2017,15 mil 779 y 2018,3 mil $330^{33}$.

Desde 1990 hasta 2013, las solicitudes de asilo nunca han fluctuado más allá de 37 mil 350 (2011), pero registraron disminuciones significativas y constantes en algunos períodos; desde 2014 ha habido un aumento importante con 63 mil 456 solicitudes, un incremento del $32 \%$ en 2015 ( 83 mil 970) y del 47\% en 2016 (123 mil 6oo $)^{34}$.

Solo en 2017 (con un aumento del 5\% en comparación con el año anterior) se registraron 130 mil 119 solicitudes de asilo, el $71 \%$ de las cuales eran de África; el 84\% eran hombres, contra el $16 \%$ de las mujeres; mientras que el grupo de edad más numeroso tenía entre 18 y 34 años, con 76\%; hay 81 mil 527 resultados de solicitudes de asilo a la Comisión Nacional de Derechos de Asilo, de los cuales el 25\% de las mujeres con solicitud de estatus de refugiado y el $29 \%$ para la protección humanitaria; el grupo de edad más registrado es entre o-13 (36\%) para el estatus de refugiado y 14-17 (63\%) para la protección humanitaria, con el 52\% de las solicitudes no reconocidas en comparación con la totalidad ${ }^{35}$.

Un aspecto del tema se puede resumir en el concepto de migración forzada, que no tiene valor legal pero que se ha utilizado en las ciencias sociales y abarca diversas interpretaciones que tienden a describir movimientos involuntarios, tanto en conflictos nacionales como externos, por las razones más dispares (desastres ambientales, hambrunas, conflictos o proyectos grupales a gran escala) y con necesidades específicas que deben ser protegidas y reconocidas internacionalmente en el acogimiento, que desafortunadamente no se materializan porque no son atribuibles a la distinción hecha por el derecho internacional entre migrante $e^{36}$ y refugiado ${ }^{37}$.

Referirse a refugiados como migrantes forzados cambia el enfoque de las necesidades específicas de los refugiados y las obligaciones legales que la comunidad internacional ha acordado sobre ellos. Para evitar este tipo de malentendidos, el ACNUR evita el uso del término migración forzada para referirse a los movimientos de refugiados y otras formas de desplazamiento (ACNUR: 2016).

En estas situaciones de desequilibrio y dificultad, la violencia, especialmente contra las mujeres, es la herramienta de dominación ampliamente utilizada: en los diversos monitoreos de organizaciones humanitarias, como las mencionadas anteriormente (en particular ACNUR: Violencia Sexual contra los Refugiados - Directrices sobre Prevención y Respuesta, Ginebra 1995), se denota cómo el fenómeno de violencia sexual es un elemento que puede encontrarse

33 Ibidem, Datos estadistico sptiembre 2018, [en linea], disponible en: http://www.libertaciviliimmigrazione.dlci. interno.gov.it/sites/default/files/allegati/settembre_2018_.

34 Ministerio del Interior Italiano, Cuaderno Estadístico por los annos 1990-2017, [en linea], disponible en: http:// www.libertaciviliimmigrazione.dlci.interno.gov.it/sites/default/files/allegati/quaderno_statistico_per_gli_ anni_1990-2017_ok.pdf

${ }^{35}$ Ibidem.

${ }^{36}$ Las personas que voluntariamente deciden abandonar los territorios nativos para mejorar sus condiciones, pero que no se caracterizan por la imposibilidad de regresar a casa de manera segura, claramente pueden solicitar asilo (derecho humano universal), pero esta distinción no terminológica podría crear confusión y desviar la atención de las medidas de protección legal específicas que requieren los refugiados.

37 Son personas que tienen que abandonar su país debido a persecución, conflicto, violencia u otras circunstancias que amenazan la paz en su país natal; se deduce que necesitan protección internacional (solicitud de asilo). El fundamento jurídico de esta protección es el art. 14 de la Declaración Universal de los Derechos Humanos el derecho de toda persona a buscar asilo y beneficiarse de él y el artículo 33 de la Convención de Ginebra de 1951 con el correspondiente Protocolo de 1967 sobre el Estatuto de los Refugiados, por el cual no pueden ser expulsados o repatriados a situaciones en las que su vida o su libertad podrían estar en riesgo. Los Estados tienen la responsabilidad primordial de proporcionar esta protección. 
antes de la decisión de emprender el desplazamiento, durante el mismo y cuando llegan a su destino: hay matrimonios-instrumentos destinados a obtener protección durante el viaje; sodomización y esclavitud a manos de las fuerzas irregulares que gestionan el flujo en los Estados limítrofes a los europeos; ataques sexuales de bandidos que bloquean los barcos que transportan a los sujetos por mar y, por último, someten las peticiones sexuales durante su estancia en los centros de acogimiento. Incluso en estos lugares, destinados al primer acogimiento y a la ayuda físico-psicológica y económica del solicitante de asilo, se crea un clima de encierro y de poca seguridad, de desconfianza frente las instituciones ${ }^{38}$.

Sujetos con cargos de autoridad que se preparan para realizar y solicitar servicios sexuales a los habitantes del campamento y, a cambio, garantizan la asistencia, la distribución de alimentos para las personas. Finalmente, incluso en el momento de la repatriación o el asentamiento en instalaciones autorizadas, encontramos abusos sexuales que dañan la identidad y la dignidad de las personas solicitantes.

Está claro que estas actitudes contra la dignidad humana se pueden encontrar en varios momentos del viaje migratorio, razón por la cual se necesita crear una plataforma de servicios (salud, psicológica, legal, administrativa) e infraestructuras (centros de acogimiento, dormitorios) más equilibrados que preserven las comunidades de origen; cuidar la provisión de dormitorios, brindando mayor seguridad y división entre las mujeres y los hombres, especialmente si han sufrido violencia durante su desplazamiento; no ingresar intempestivamente a los solicitantes a la turbina de los roles e ideales de género de la sociedad de acogida, ya que podrían crear confusión y pérdida tanto para las personas (víctimas) como para aquellos que siempre han vivido en un entorno cultural en el que la identidad de genero tiene un papel diferente (evitando que las personas del sexo opuesto se sientan limitadas por el nuevo entorno social y el papel que puedan tener, creando situaciones de violencia y de inseguridad). Es importante organizar capacidades de información y de actualización para ayudar a los voluntarios de los centros, pero también a los ciudadanos de los lugares donde se crean estas realidades para una mayor cohesión y una capacidad de gestión real.

Un ejemplo relativamente reciente, un símbolo de cómo los conflictos étnicos pueden explotar la esfera privada y corporal de las mujeres es lo que sucedió en Nigeria que, desde 2009 se caracterizó por una fuerte presión militar y política de origen terrorista del grupo extremista Boko Haram. Esta entidad asociativa se interpreta de varias maneras: desde el repudio de las enseñanzas occidentales hasta la propaganda de las enseñanzas del profeta Mahoma, pero, independientemente de la conexión religiosa, el movimiento nunca se caracterizó por actos violentos, sino que nació como una realidad sociopolítica a favor de la igualdad social que en el Estado estaba excluida de actos de política corrupta y discriminatoria.

Un hecho emblemático, que explica el cambio en la acción del grupo es el secuestro de 219 niñas el 14 de abril de 2014, en una escuela secundaria en la ciudad de Chibok (otro caso de vio-

\footnotetext{
${ }^{38}$ En 2017, el ACNUR registró a 622 sobrevivientes de violencia sexual en las islas griegas del Egeo; en particular, el $28 \%$ de estas experiencias tuvieron lugar después de su llegada a los Centros de Recepción e Identificación: $L a$ situación es particularmente preocupante en los Centros de Recepción e Identificación (RIC) de Moria (Lesbos) y Vathy (Samos), donde miles de los refugiados siguen permaneciendo en refugios inadecuados con seguridad inadecuada. Alrededor de 5.500 personas se encuentran en estos centros, que están al doble de su capacidad. Los informes de acoso sexual en Moria son particularmente altos. UNHCR (9 febrero 2018), Refugee women and children face heightened risk of sexual violence amid tensions and overcrowding at reception facilities on Greek islands, [en linea], disponible en: http://www.unhcr.org/news/briefing/2018/2/5a7d67c4b/refugee-women-children-faceheightened-risk-sexual-violence-amid- tensions.html.
} 
lencia de género y explotación del cuerpo femenino) pero hay muchos actos de violencia contra los vulnerables: según el Informe 2017-2018 de Human Rights Watch:

Boko Haram usó principalmente mujeres y niñas como kamikaze, obligándolos a explotar bombas en centros urbanos. Según el Fondo de las Naciones Unidas para la Infancia, UNICEF, 83 niños han sido utilizados como terroristas suicidas desde enero: 55 niñas y 27 niños, uno era un niño atado a una niña. El grupo secuestró a 67 mujeres y niños en 2017. El conflicto de [...] ocho años de Nigeria con Boko Haram ha matado a más de 20,0oo civiles y una crisis humanitaria a gran escala. Alrededor de 2.1 millones de personas han sido desplazadas por el conflicto (la mayoría huye al vecino Camerún y la canaliza a los centros de refugiados), mientras que 7 millones necesitan asistencia humanitaria.

Al mismo tiempo, el informe de EASO (Oficina Europea de Apoyo al Asilo) de junio de 2017 calcula

El $28 \%$ de las mujeres nigerianas entrevistadas entre las edades de 15 y 40 años sufrieron violencia física al menos una vez a la edad de 15 años y el 11\% la experimentó el año anterior a la encuesta. De las mujeres que nunca han estado casadas, el $25 \%$ ha sufrido abuso físico, sexual o emocional en el pasado, el 19\% lo ha experimentado en el último año. En comparación, el $35 \%$ de las mujeres solteras, el $30 \%$ de las mujeres sin hijos y el $42 \%$ divorciadas, mujeres separadas o viudas informaron haber sido violentas desde la edad de 15 años. En general, el $7 \%$ de las mujeres de 15 a 49 años de edad sufrieron violencia sexual al menos una vez, con una mayor proporción proveniente de grupos católicos y cristianos y entre mujeres divorciadas, separadas o viudas. La violación es común y generalizada el estigma social reduce la probabilidad de que las víctimas lo denuncien o que los autores sean perseguidos o castigados ${ }^{39}$.

El fenómeno está estrechamente relacionado con la marginación de los sujetos femeninos, las situaciones de pobreza en que viven, su subordinación a las costumbres locales y la voluntad del jefe de familia que otorga más importancia a la preservación del honor del clan que a la dignidad humana ${ }^{40}$.

Incluso con la aprobación en 2015 de la VAPP (Ley de Prohibición de Violencia Contra las Personas), que tipifica como delito la violación y aborda el problema de la violencia sexual, física y psicológica, prohíbe las prácticas tradicionales nocivas, considera como un delito la paliza del cónyuge, la expulsión forzosa de la casa, los abusos económicos coercitivos ${ }^{41}$, los actos de violen-

${ }^{39}$ NPC/ICF Int., NDHS 2013, 2014, Chapter 16.

${ }^{40}$ Hay varios ejemplos que denotan esta fragilidad social: la práctica de la mutilación genital femenina (mutilación de genitales femeninos) afecta a varios Estados africanos y se basa en la creencia de que el contacto con el clítoris y la cabeza del bebé durante el parto es letal y perjudicial, así como por una razón de higiene y preservación de la fertilidad y feminidad de las mujeres (según la encuesta NDHS de 2013, un 24,8\% de todas las mujeres entre las edades de 15 y 49 años fueron mutiladas), el matrimonio forzado, a una edad temprana, por razones económicas ( $50 \%$ de las niñas nigerianas menores de 18 años); el tráfico de personas para la prostitución dentro y hacia países europeos (en la ciudad de Benin, una ciudad nigeriana famosa por este intercambio comercial, hay varias asociaciones criminales que patrocinan los viajes en Europa a las niñas que, al llegar a su destino, tendrán que pagar a sus líderes a través de la explotación sexual).

${ }^{41}$ Easo (2017), Country of Origin Information Report. Nigeria, [en linea], disponible en: https://coi.easo.europa.eu/ administration/easo/PLib/EASO_Country_Focus_Nigeria_June2017.pdf pp. 38. 
cia no disminuyen sino que crean inseguridad hacia el Estado y la necesidad de abandonar el lugar de origen.

En relación con los conflictos internos ejercidos por las milicias de Boko Haram, en 2014, en Italia, hubo 10 mil 040 solicitudes de asilo ${ }^{42}$, con un incremento anual constante (18 mil 174 en 2015, 27 mil 289 en 2016) hasta un ligero descenso ( $5 \%$ con 25 mil 964) ${ }^{43}$ en 2017 y un crecimiento del $40 \%$ entre agosto y septiembre de 2018 (de 212 a 296$)^{44}$.

A partir de estas circunstancias diarias, una intervención legislativa se ha vuelto esencial para limitar y abordar los fenómenos de la mala gestión de la entrada en los Estados involucrados: a nivel nacional ${ }^{45}$ y supranacional ${ }^{46}$, durante los últimos veintiocho años, se han realizado varias maniobras legislativas para promover y apoyar los derechos de los inmigrantes y el desarrollo de su integración en los Estados de acogida, a fin de desalentar cualquier forma de discriminación. Pero el proceso aún es muy difícil y está lleno de trampas ideológicas y de explotación. El viaje a la costa europea es muy arriesgado: según la encuesta del ACNUR, del 6 de agosto de 2018:

${ }^{42}$ Ministerio del Interior Italiano, Cuaderno Estadístico por los annos 1990-2017, [en linea], disponible en: http://www.libertaciviliimmigrazione.dlci.interno.gov.it/sites/default/files/allegati/quaderno_statistico_per_ gli_anni_1990-2017_ok.pdf

43 Ministerio del Interior Italiano, Datos estadistico 2017, [en linea], disponible en: http://www.libertaciviliimmigrazione.dlci.interno.gov.it/sites/default/files/allegati/dati_asilo_2017_.pdf

44 Ibidem, Tablero estadístico diario 23-10-2018, [en linea], disponible en: http://www.libertaciviliimmigrazione. dlci.interno.gov.it/sites/default/files/allegati/cruscotto_statistico_giornaliero_23-10-2018

${ }^{45}$ Ley n. 39 / 1990 (Ley Martelli) que sancionó la planificación cuantitativa de los flujos migratorios, Ley n. 40 / 1993 (Ley Mancino) y el Decreto Dini de 1995; Parlamiento Italiano, Ley 40/1998 (Ley Napolitano), [en linea], disponible en: http://www.camera.it/parlam/leggi/9804ol.htm, con la introducción del Texto Consolidado sobre Inmigración, que regula con mayor precisión el marasmo legislativo y le da vida al CPT (Centros de Estancia Temporal: un mes máximo, CIE futuro, Centros de Identificación y expulsión máxima de 6 meses); PARLAmiento ItAliAno, Ley n. 189/ 2002 (Ley Bossi-Fini), [en linea], disponible en: http://www.camera.it/parlam/leggi/o2189l. htm, de matriz intransigente y oclusiva; Parlamiento Italiano, Ley n. 125 / 2008, [en linea], disponible en: http://www.camera.it/parlam/leggi/o8125l.htm, que introduce nuevos tipos de delitos para los inmigrantes ilegales y aquellos que favorecen la entrada. Gazzetta Ufficiale de la Republica Italiana, Ley n. 94 / 2009, [en linea], disponible en: http://www.gazzettaufficiale.it/gunewsletter/dettaglio.jsp?service $=1 \&$ datagu=2009-0724\&task=dettaglio\&numgu=170\&redaz $=009$ Goo96\&tmstp $=1248853260030 ;$ Ibidem, Ley $n .119 / 2013$, [en linea], disponible en: http://www.gazzettaufficiale.it/eli/id/2013/10/15/13Goo163/sg.

Sobre seguridad y contraste de la violencia de género con la posibilidad de emitir un permiso de residencia especial para las víctimas de violencia que también sufrieron durante el alojamiento en los centros de recepción. Decreto Legislativo n. 24 / 2014 que extiende este instrumento a las víctimas de delitos de reducción y mantenimiento en esclavitud y trata de personas. Ibidem, Ley 46 / 2017, [en linea], disponible en: http://www.gazzettaufficiale.it/ eli/id/2017/04/18/17Goo059/sg, que establece las reglas diseñadas para acelerar los procedimientos relativos a la protección internacional.

${ }^{46}$ Parlamento Europeo y Consejo de la Union Europea, Directiva “Calificaciones” 2011/95 / UE, [en linea], disponible en: https://web.icam.es/bucket/DIRECTIVA\%2095\%202011\%20CALIFICACION\%20Loooo9-0oo26. pdf que normaliza a los distintos estados para disfrutar de protección internacional; Ibidem, Directiva "procesal" 2013/32 / UE, [en linea], disponible en: http://www.acnur.org/fileadmin/Documentos/BDL/2013/926o.pdf , que regula el proceso de solicitud de asilo; Ibidem, Directiva "recepción" 2013/33 / UE, [en linea], disponible en: https://eur-lex.europa.eu/legal-content/ES/TXT/PDF/?uri=CELEX:32013Loo33\&rid=1, se refiere al acceso a las condiciones de recepción para solicitantes de asilo en espera de su solicitud; Ibidem, Reglamento de Dublín (604/2013), [en linea], disponible en: https://eur-lex.europa.eu/legal-content/ES/TXT/PDF/?uri=CELEX:32013Ro $604 \&$ rid=1, que indica la responsabilidad de examinar la solicitud al Estado miembro que ha desempeñado el papel principal en relación con Admisión o residencia del solicitante; Ibidem, Reglamento "Eurodac" (603/2013), [en linea], disponible en: https://eur-lex.europa.eu/legal-content/ES/TXT/?uri=OJ:L:2013:18o:TOC, Sistema de control y comparación de huellas dactilares. 
Más de 1,500 refugiados y migrantes perdieron la vida tratando de cruzar el Mediterráneo en los primeros siete meses de 2018. [...] Aproximadamente 6o,ooo personas cruzaron el Mediterráneo este año, la mitad en comparación con el mismo período de 2017, lo que marca un regreso a niveles previos a 2014. Sin embargo, cada 31 personas que intentaron cruzar en junio y julio, una está muerta o desaparecida, en comparación con 1 en 49 en 2017.

Los traficantes que organizan estos viajes de esperanza explotan continuamente la necesidad y la desesperación de los migrantes, sin tener en cuenta los estándares básicos de seguridad para garantizar un viaje de este tipo. Y cuando ocurren desgracias, los Estados actúan con negligencia y miedo ${ }^{47}$, con pasajes hipócritas y contrarios a las normas del derecho internacional y humanitario.

Las relaciones de subordinación y sujeción se crean sistemáticamente entre el sujeto que ofrece el viaje y los que lo necesitan; entre aquellos que tienen el poder de elegir las condiciones de partida (transporte, ruta, precio y, por consiguiente, limitación de la libertad) y aquellos que inevitablemente sufren en silencio la necesidad de tomar tales decisiones con miras a la llegada y la salvación. La trata de seres humanos. Por sí sola hace que esta relación de miedo y esclavitud, en su mayoría de naturaleza sexual, se perpetúe, incluso después del desembarco en el país de la UE.

Del análisis completo es lógico concluir que la violencia se convierte en una fuerza social y no solo en el poder o su instrumento, sino que reclama la conciencia de los sujetos que operan en una trascendencia mistificada de sí mismos y del grupo al que pertenecen; es la regeneración perversa de sí mismo para perpetuarlo hasta la concreción del ideal mismo. Dicha acción tiene como origen el sentido psicológico y social de la privatización de las expectativas: cuanto más intensa es la frustración, mayor es la intensidad del conflicto y de la fuerza violenta. Cuanto mayor es la dificultad social, mayor será la búsqueda en la estructura social para encontrar un elemento común que lo una e identifique (identidad de grupo).

El poder de exponer a una población a una muerte general es el otro lado del poder para garantizar a otro su mantenimiento de la existencia. El principio: poder matar para poder vivir, que apoyaba las tácticas de los combatientes, se convirtió en el principio de estrategia entre los estados; pero la existencia en cuestión ya no es la jurídica de soberanía, sino la biológica de una población. Si el genocidio es el sueño de los poderes modernos, es porque el poder se coloca y se practica a nivel de la vida, las especies, la raza y los fenómenos de la población masiva ${ }^{48}$.

Los conceptos de validez, vinculación y efectividad de la norma legal son suplantados por una respuesta descuidada de la sociedad, una orientación social que se caracteriza por una estructura vacía de demandas y expectativas y, en consecuencia, la ley no puede crear oportunidades para comprender cómo se identifican los principios fundamentales y su cambio en el sentido común. La comunicabilidad de los mensajes normativos es fundamental, pero se ve afectada por las formas en que se da a conocer a la sociedad y esta responde con actos no conformes.

El poder de las normas para afectar la esfera individual está inevitablemente conectado a su incapacidad para tener en cuenta cómo las variables económico-sociales del neoliberalismo actual han llevado a un empobrecimiento del significado de salvaguardia colectiva, en favor de una mayor individualización. El simbolismo del lenguaje en contextos de conflicto y de inter-

\footnotetext{
47 El caso de los barcos de "Acuario", con 629 migrantes a bordo y "Lifeline" de junio de 2018. UNHCR, Superó el umbral de 1.500 muertes en el Mediterráneo o6/o8/2018, [en linea], disponible en: https://www.unhcr.it/news/ unhcr-superata-la-soglia-1-50o-morti-nel-mediterraneo.html

${ }^{48}$ Foucault, Michel (1976), La voluntad de saber, Feltrinelli Editore, Roma, p. 121.
} 
vención humanitaria es poderoso y está privado de ese papel histórico y concreto que caracteriza el motivo mismo de las intervenciones internacionales, devolviéndolos a las prácticas burocráticas y de sentirse cómodo.

Los derechos humanos se han convertido ahora en el instrumento de dominación y el supuesto de la ayuda humanitaria actual es ayudar a los sujetos locales que no pueden resolver las situaciones de gestión interna, reduciendo todo en un retorno a lo colonial. Prácticas administrativas externas al contexto específico. La idea básica es una especie de constitucionalismo global que surge en una relación de hegemonía de los valores occidentales y del lenguaje generalizado y común de la disidencia y de la revuelta hacia una globalización económica y militarizada que produce, junto con la opresión y la macroscopia desigualdades, guerras y conflictos ${ }^{49}$.

En mi opinión, una respuesta irrazonable a la norma jurídica es el resultado de una disfunción estructural de la sociedad actual: la violencia, y en particular la de género, sigue siendo una construcción social en un sentido objetivo y subjetivo; el temor por el migrante y por lo desconocido es evidente; la falta de asistencia social del Estado sigue siendo tal y la desigualdad continúa extendiéndose. La enfermedad es interna a la sociedad y el Estado es su reflejo.

Se necesitan políticas y acciones intersectoriales concretas de valor integracionalista: la información y la participación de la comunidad en situaciones grupales, principalmente mujeres, son fundamentales para crear ese corolario de las relaciones sociales, la equidad y la usabilidad de los recursos estatales.

La comprensión de cómo la esfera de género siempre ha sido un elemento detonante para la formación de problemas sociales es consecuente con la explicación de cómo, aún hoy, las mujeres siguen siendo, la mayoría de la veces por razones culturales, entidades subjetivas vulnerables que deben ser protegidas, tratando con procesos de información básicos (salud, autodeterminación, educación). Solo será posible con estos medios de conciencia crear una colaboración social completa y, de hecho, ser capaz de aceptar, sin monólogos políticos delirantes, el otro, que no es diferente pero es NOS.

Con la eliminación de la violencia estructural, inherente a la sociedad, que describe a algunos sujetos como vulnerables simplemente porque los extranjeros, las mujeres, los menores y los pobres carecen de una fuerza de acción concreta y cotidiana, de una voz real igual a un rango de población incongruentemente más interesante, habrá igualdad y respeto al entrar en contacto con la esfera pública.

\section{Referencias bibliográficas:}

Amnesty International, Report 2017/2018 Serbia, [en linea], disponible en: https://www.amnesty.it/rapporti-annuali/rapporto-annuale-2017-2018/europa/serbia/

, Rwanda, [en linea], disponible en: https://www.amnesty.it/rapporti-annuali/rapporto-annuale-2017-2018/africa/ruanda/

Asamblea General de las Naciones Unidas (1979), CEDAW, [en linea], disponible en: http://dirittiumani.donne.aidos.it/bibl_2_testi/b_patti_conv_protoc/a_testi_7_conv_ pricip/d_cedaw_donne/home_cedaw.html

\footnotetext{
${ }^{49}$ CASADEI, Thomas (2006), Ética práctica y casos de guerra: la parafernalia argumentativa de Michael Walzer, [en linea], disponible en: http://www.juragentium.org/topics/wlgo/it/casadei.htm
} 
(1984), Convención contra la Tortura y otros tratos o penas crueles, inhumanos y degradantes, [en linea], disponible en: https://www.unhcr.it/wp-content/uploads/2016/o1/ Convenzione_contro_la_Tortura.pdf

(1951), Convención de Genevra, [en linea], disponible en: https://www.unhcr.it/wpcontent/uploads/2016/o1/Convenzione_Ginevra_1951.pdf

(1948), Declaración universal de los derechos humanos, [en linea], disponible en: https:// www.ohchr.org/EN/UDHR/Documents/UDHR_Translations/itn.pdf

Butler, Judith (2013), Cuestión de género. El feminismo y la subversión de la identidad, Laterza, Roma.

(2009), Sujetos del deseo, Laterza, Roma

(2006), La derrota del género, Meltemi, Parma.

(2006), Crítica de la violencia ética, Feltrinelli, Milano.

(1996), Cuerpos que cuentan. Los límites discursivos del "sexo", Feltrinelli, Milano.

CASAdeI, Thomas (2006), Ética práctica y casos de guerra: la parafernalia argumentativa de Michael Walzer, [en linea], disponible en: http://www.juragentium.org/topics/wlgo/it/ casadei.htm

CASADEI, Thomas (2012), Derechos humanos y personas vulnerables. Violaciones, transformaciones, aporias, Giappichelli Editore, Torino.

Consejo DE Europa (2007), Convención de Lanzarote, [en linea], disponible en: https://www. garanteinfanzia.org/sites/default/files/documenti/2013-11-18_Convenzione\%2oLanzarote.pdf

(2011), Convención de Istambul, [en linea], disponible en: https://www.senato.it/japp/ bgt/showdoc/17/DOSSIER/o/750635/index.html?part=dossier_dossier1-sezione_sezione2-h2_h22

(1950), CEDU, [en linea], disponible en: https://www.echr.coe.int/Documents/Convention_ITA.pdf

Consejo de Seguridad de las Naciones Unidas, Resoluciòn n1973/1975, [en linea], disponible en: https://eur-lex.europa.eu/LexUriServ/LexUriServ.do?uri=OJ:C:1975:179:FULL:IT :PDF

, n.1325/200o, [en linea], disponible en: http://www.un.org/en/ga/search/view_doc. asp?symbol=S/RES/1325\%282000\%29

, n.1820/2008, [en linea], disponible en: https://documents-dds-ny.un.org/doc/UNDOC/GEN/No8/391/44/PDF/No839144.pdf?OpenElement 
, n.1888/2009, [en linea], disponible en: https://documents-dds-ny.un.org/doc/UNDOC/GEN/No9/534/46/PDF/No953446.pdf?OpenElement

,n.1960/2010, [en linea], disponible en: https://documents-dds-ny.un.org/doc/UNDOC/ GEN/N10/698/34/PDF/N1069834.pdf?OpenElement

, n.2106/2013, [en linea], dispomible en: https://documents-dds-ny.un.org/doc/UNDOC/GEN/N13/372/15/PDF/N1337215.pdf?OpenElement

, n.2122/2013, [en linea], disponible en: https://www.un.org/press/en/2013/sc11149.doc. htm

, n.2242/2015, [en linea], disponible en: https://undocs.org/S/RES/2242(2015)

Corte Costitutional Italiana (1948), Constitución Italiana, [en linea], disponible en: https://www.cortecostituzionale.it/documenti/download/pdf/Costituzione_della_Repubblica_italiana.pdf

Corte Penal Internacional (1998), Estatuto de Roma, [en linea], disponible en: http://www. cirpac.it/pdf/testi/Statuto\%2odi\%2oRoma\%2odella\%2oCorte\%2oPenale\%2oInternazionale.pdf

Chrétien, Jean-Pierre (1977), Les deux visages de Cham. Points de vue français du XIXe siècle sur les races africaines d'après l'exemple de l'Afrique orientale, in GuIRAL, P. y TemI$\mathrm{NE}$, E., L'idée de race dans la pensée politique française contemporaine, Paris, C.N.R.S., française, Editions du CNRS, pp. 177- 99.

De Beauvoir, Simone (1946), El segundo sexo, trad. Roberto Cantini y Mario Andreose, Il Saggiatore, Milán, 1961.

Di Palma, Sara Valentina (2010), La violación como arma contra las mujeres: la ex Yugoslavia, Ruanda y el área de los grandes lagos africanos, Eds. Flores, Marcello, Los raptos de guerra. Violencia masiva contra las mujeres en el siglo XX. Franco Angeli. Milano, p. 218.

EASO, (junio 2017), Country of Origin Information Report. Nigeria, [en linea], disponible en: https://coi.easo.europa.eu/administration/easo/PLib/EASO_Country_Focus_Nigeria_ June2017.pdf pp.34-38-42-45.

Foucault, Michel (1976), La voluntad de saber, Feltrinelli Editore, Roma.

Friedan, Betty (2014), La mística de la feminidad, Castelvecchi, Roma.

Fusaschi, Michela (200o), Hutu-tutsi. En los orígenes del genocidio ruandés, Boringhieri, Torino.

(2011), Cuando el cuerpo es de los demás. Retórica de piedad y humanitarismo-show, Boringhieri, Torino.

Galimberti, Umberto (2002), El cuerpo, Feltrinelli, Milan. 
Gazzetta Ufficiale de la Republica Italiana, Código Civil Italiano. 1942 aprobado con Decreto Real el 16 de marzo de 1942, n. 262, [en linea], disponible en: http://www.jus. unitn.it/cardozo/Obiter_dICTUM/codciv/Codciv.htm

, Ley n.989/1970, [en linea], disponible en: http://www.gazzettaufficiale.it/eli/ $\mathrm{id} /$ 1970/12/o3/o7oUo898/sg;jsessionid=Y2ITexX-cjfTXYBT4MhBqQ_.ntc-as3-guri2b

, Ley n.194/1978, [en linea], disponible en: http://www.gazzettaufficiale.it/atto/serie_generale/caricaDettaglioAtto/originario?atto.dataPubblicazioneGazzetta=1978-0522\&atto.codiceRedazionale $=078$ U0194\&elenco3ogiorni $=$ false

, Ley n.39/199o, [en linea], dispoible en: http://www.gazzettaufficiale.it/eli/ $\mathrm{id} / 1990 / 02 / 28 / 090 G 0075 / \mathrm{sg}$

, Ley n. 40/2004, [en linea], disponible en: http://www.gazzettaufficiale.it/eli/ $\mathrm{id} / 2017 / 02 / 17 / 17 \mathrm{GooO} 24 / \mathrm{sg}$

, Ley n. 94/2009, [en linea], disponible en: http://www.gazzettaufficiale.it/gunewsletter/ dettaglio.jsp? service $=1 \&$ datagu $=2009-07-24 \&$ task $=$ dettaglio $\&$ numgu $=170 \&$ redaz $=009$ Goo96\&tmstp $=1248853260030$

, Ley $n$. 119/2013, [en linea], disponible en: http://www.gazzettaufficiale.it/eli/ $\mathrm{id} / 2013 / 10 / 15 / 13 \mathrm{GoO} 163 / \mathrm{sg}$

, Ley n. 46/2017, [en linea], disponible en: http://www.gazzettaufficiale.it/eli/ $\mathrm{id} / 2017 / 04 / 18 / 17 \mathrm{Goo0} 59 / \mathrm{sg}$

, Ley n.219/2017, [en linea], disponible en: http://www.gazzettaufficiale.it/eli/ $\mathrm{id} / 2018 / 1 / 16 / 18 \mathrm{Goooo6} / \mathrm{sg}$

, Código Penal Italiano. 1930 aprobado con Decreto Real el 19 de octubre de 1930, n. 1398, [en linea], disponible en: https://www.altalex.com/documents/codici-altalex/2014/10/30/ codice-penale

Gellately, Robert y Kiernan, Ben (eds. 2006), El siglo del genocidio, Longanesi, Milan.

Human Rights Watch, Report 2018 Nigeria, [en linea] disponible en: https:/www.hrw.org/ world-report/2018/country-chapters/nigeria

HuSSERL, Edmund (1999), Meditaciones cartesianas. Introducción a la fenomenología, Kluwer Academic Publishers, Berlin.

Irigaray, Luce (2010), Speculum. De la otra mujer, Feltrinelli, Milan.

Liga de estados arabes, Carta Árabe de Derechos Humanos 1994, [en linea], disponible en: http://hrlibrary.umn.edu/instree/loas2005.html

Kristeva, Julia (2007), La revolución del lenguaje poético, Spirali, Milan.

MaCKinnon, Catharine (2012), ¿Son las mujeres humanas?, Laterza, Roma. 
(1989), Towards a Feminist Theory of the State, Harvard University Press, Cambridge.

MEAD, Margaret (2009), Sexo y temperamento en tres sociedades primitivas, Il saggiatore, Milan.

(1972), Hombre y mujer, Il saggiatore, Milan.

Millett, Kate (1970), Sexual politics, Rizzoli, Milan.

Ministerio del Interior Italiano, Cuaderno Estadístico por los annos 199o-2017, [en linea], disponible en: http://www.libertaciviliimmigrazione.dlci.interno.gov.it/sites/default/files/allegati/quaderno_statistico_per_gli_anni_1990-2017_ok.pdf

Datos estadistico 2017, [en linea], disponible en: http://www.libertaciviliimmigrazione.dlci.interno.gov.it/sites/default/files/allegati/dati_asilo_2017_.pdf

, Datos estadistico settembre 2018, [en linea], disponible en: http://www.libertaciviliimmigrazione.dlci.interno.gov.it/sites/default/files/allegati/settembre_2018_.pdf

, Tablero estadístico diario 23-10-2018, [en linea], disponible en: http://www.libertaciviliimmigrazione.dlci.interno.gov.it/sites/default/files/allegati/cruscotto_statistico_giornaliero_23-10-2018.

Nussbaum, Martha C. (2000), Sex and Social Justice, Oxford University Press, New York.

OUA (1986), Carta Africana de Derechos Humanos y de los Pueblos, [en linea], disponible en: https://au.int/en/treaties/african-charter-human-and-peoples-rights

Parlamento Europeo y Consejo de la Union Europea, Directiva $n$. 2011/95/UE, [en linea], disponible en: https://web.icam.es/bucket/DIRECTIVA\%2095\%202011\%20CALIFICACION\%20Loooo9-00026.pdf

, Directiva n. 2013/32/UE, [en linea], disponible en: http://www.acnur.org/fileadmin/ Documentos/BDL/2013/926o.pdf

, Directiva n. 2013/33/UE, [en linea], disponible en: https://eur-lex.europa.eu/legal-content/ES/TXT/PDF/?uri=CELEX:32013Loo33\&rid=1

, Reglamento n. 603/2013, [en linea], disponible en: https://eur-lex.europa.eu/legal-content/ES/TXT/?uri=OJ:L:2013:180:TOC

, Reglamento n. 604/2013, [en linea], disponible en: https://eur-lex.europa.eu/legal-content/ES/TXT/PDF/?uri=CELEX:32013Ro6o4\&rid=1

Parlamento Italiano, Ley $n$. 40/1998, [en linea], disponible en: http://www.camera.it/parlam/leggi/9804ol.htm

Ley n.91/1999, [en linea], disponible en: http://www.camera.it/parlam/leggi/99091l. htm 
, Ley n. 189/2002, [en linea], disponible en: http://www.camera.it/parlam/leggi/o2189l. htm

, Ley n.125/2008, [en linea], disponible en: http://www.camera.it/parlam/leggi/o8125l. htm

, Ley n. 119/2013, [en linea], disponible en: http://www.gazzettaufficiale.it/eli/ $\mathrm{id} / 2013 / 10 / 15 / 13 \mathrm{GoO} 163 / \mathrm{sg}$

, Ley n. 46/2017, [en linea], disponible en:. http://www.gazzettaufficiale.it/eli/ id/2017/04/18/17Gooo59/sg

, Ley n.219/2017, [en linea], disponible en: http://www.gazzettaufficiale.it/eli/ $\mathrm{id} / 2018 / 1 / 16 / 18 \mathrm{Goooo} 6 / \mathrm{sg}$

Pitch, Tamar (1995), La antropología de los derechos humanos, en A. Giasanti, G. Maggioni, (editado por), Los derechos ocultos. Enfoque antropológico y perspectiva sociológica, Cortina, Milán.

(1998), Un derecho para dos. La construcción legal de género, sexo y sexualidad, Il Saggiatore, Milán.

(2012), Contra el decoro. El uso político de la decencia pública, Laterza, Roma.

Rivera, Annamaria (2010), Lo bello, la bestia y lo humano, Ediesse, Roma.

(2015), Violación de la guerra y violencia de género, Ediesse, Roma.

Rodotà, Stefano y Zatti, Paolo (eds. 2011), Tratado de biodiritto. El gobierno del cuerpo (vol.III), Giuffrè Editore, Milan.

Tribunal Constitucional Italiano, Sentecia n.221/2015, [en linea], disponible en; https:// www.cortecostituzionale.it/actionSchedaPronuncia.do? $a n n o=2015 \&$ numero $=\mathbf{2 2 1}$

Tribunal de Roma, Sentencia n.2049/2007, [en linea], disponible en: http://www.biodiritto. org/index.php/item/849-welby

UNHCR (1993), Refugee Protection and Sexual ViolenceRefugee Protection and Sexual Violence No. 73 (XLIV), 01/10/1993, [en linea], disponible en: http://www.unhcr.org/excom/ exconc/3ae68c6810/refugee-protection-sexual-violence.html

(2016), Refugiados y migrantes, 21/04/2016, [en linea], disponible en: https://www.unhcr.it/news/rifugiati-e-migranti-faqs.html

(2000), El genocidio ruandés y sus consecuencias, pp. 248-256, [en línea], disponible en: http://www.unhcr.org/pubs/sowr20oo/italian/ch1o.pdf

(2018), Amnesty International Report 2017/2018 Somalia, [en linea], disponible en: http://www.refworld.org/docid/5a99386aa.html 
(2017), Report Rwanda 2017, p. 6, [en linea], disponible en: http://www.unhcr.org/rw/ wp-content/uploads/sites/4/2018/o1/PA-2017-REPORT.pdf

(2018), Refugee women and children face heightened risk of sexual violence amid tensions and overcrowding at reception facilities on Greek islands, 09/03/2018, [en linea], disponible en: http://www.unhcr.org/news/briefing/2018/2/5a7d67c4b/refugee-womenchildren-face-heightened-risk-sexual-violence-amid-tensions.html

(2018), Superó el umbral de 1.500 muertes en el Mediterráneo o6/o8/2018, [en linea], disponible en: https://www.unhcr.it/news/unhcr-superata-la-soglia-1-50o-morti-nelmediterraneo.html

United Nations, International Criminal Tribunal for the former Yugoslavia, [en linea], disponible en: http://www.icty.org/ , International Criminal Tribunal for Rwanda, [en linea], disponible en: http://unictr. irmct.org/

Witting, Monique (1976), El cuerpo lésbico, Edizioni delle Donne, Roma. 